Or, en rapprochant ces remarques des constatations que nous a vons faites a près administration à la vache d'huile de foie de morue (coloration du lait et du beurre augmentée, poids de matière grasse plus élevé), et a près ingestion de carottes (coloration du beurre plus intense et goût plus agréable), nous avons pensé qu'il y aurait intérêt, pour la santé des enfants, pour leur développement et leur croissance, à donner des laits riches en vitamines.

L'expérience semble confirmer nos observations. Nous a vons fait a bsorber quotidiennement à des vaches, a vec leur ration habituelle, $3 \mathrm{~kg}$. de carottes et une dose de 150 à $200 \mathrm{gr}$. d'huile de foie de morue. Une jeune mère, dont le mari est domestique au Fieu, ayant sevré son enfant, a donné, pendant l'hiver 1931-1932, ce lait supposé ainsi survitaminé. Nous avons constaté que la santé de l'enfant a été parfaite pendant toute cette période ; sa taille et son poids ont normalement augmenté.

Nous croyons devoir signaler ces observations pour le plus grand intérêt de la théra peutique infantile : la pratique des substitutions alimentaires des grandes femelles laitières deviendrait une nécessité.

\title{
L'ACIDE PICRIQUE DANS LE LAIT. SA CARACTÉRISATION
}

\author{
par M. LAGRANGE-FRANCÈS
}

Docteur en pharmacie.

En opérant sur plusieurs animaux, nous avons constaté que lorsqu'on administre à une vache des doses eroissantes d'acide picrique, il arrive un stade où le lait prend un aspect jaunâtre et garde cette teinte-limite quelle que soit la quantité d'acide picrique ingérée en excès.

L'apparence du lait est ainsi très favorable; sa couleur peut en effet laisser supposer qu'il est très riche en beurre:

Son odeur n'est pas modifiée. Il ne présente aucune saveur anormale, contrairement à ce que l'on aurait pu croire. Un enfant l'accepte très volontiers. L'acidité n'est pas augmentée.

On ne note aucune variation appréciable dans les éléments normaux du lait.

Or, si un fraudeur ajoute de l'eau à un lait normal, ce lait prend une teinte bleutée caractéristique. Avec un lait picriqué, au contraire, l'addition d'eau passe inaperçue en apparence : l'aspect du lait reste favorable, et le consommateur non averti ne peut soupçonner la fraude à simple vue.

Bien qu'à notre connaissance, cette fraude n'ait jamais été pratiquée, il nous a paru intéressant d'étudier comment on pourrait 
la dépister par la caractérisation de l'acide picrique. Nous avons opéré soit avec des laits directement picriqués, soit avec des laits de vaches ayant absorbé des quantités d'acide picrique variant de 1 à 12 grammes.

- Nous avons naturellement pensé à earactériser l'acide picrique par la réaction classique du Rupeau-Le Mithouard sur le liquide obtenu par désalbumination du lait, soit par l'acide métaphosphorique, soit par l'acide trichloracétique. Nous nous sommes heurté à une impossibilité absolue, même avec les laits direetement additionnés d'acide plerique.

C'est alors que nous nous sommes souvenu d'une curieuse observation de Конn-Aвrest. Si l'on essaie d'extraire par le chloroforme l'acide picrique d'une solution aqueuse contenant de l'acide tartrique, on échoue à coup sûr. On réussit, au contraire, si l'on remplace l'acide tartrique par l'acide sulfurique.

Nous avons émis l'hypothèse que l'acide tartrique pouvait bien avoir une action empêchante du fait de la présence des oxhydriles alcooliques contenus dans sa molécule. S'il en est ainsi, nos échecs sur le lait pouvaient bien provenir de la présence du lactose, encore plus riche en oxhydriles que l'acide tartrique, et si on détruisait le sucre, on devait alors réussir.

L'expérience est venue confirmer cette façon de voir. Pour éliminer le lactose, il suffit de faire agir sur lui un mélange de sulfate de cuivre et de chaux, comme on le fait pour détruire le glucose des urines où l'on veut doser l'acide $\beta$-oxybutyrique.

Après de multiples essais, nous avons arrêté la technique suivante :

Dans une fiole eonique, verser $25 \mathrm{~cm}^{3}$ de lait, $50 \mathrm{~cm}^{3}$ de solution de sulfate de cuivre à $35 \mathrm{gr}$. par litre, $5 \mathrm{~cm}^{3}$ d'eau environ et $50 \mathrm{~cm}^{3}$ de lait de chaux fraîchement préparé.

Agiter ; laissez au moins 1 heure en agitant fréquemment.

Filtrer. Si les premières gouttes recueillies ne sont pas alcalines à la phtaléine du phénol, ajouter encore un excès de lait de chaux, pour que le filtrat soit alcalin.

On obtient ainsi un filtrat généralement jaune clair et ne contenant plus de glucides réducteurs.

Le liquide est concentré à environ $100 \mathrm{~cm}^{3}$ par ébullition et de nouveau filtré.

Prélever $50 \mathrm{~cm}^{3}$ de liquide dans une boule à décantation et ajouter $5 \mathrm{~cm}^{3}$ d'un mélange de 1 volume d'éther pour 2 volumes de chloroforme. Agiter. Décanter et filtrer le liquide chloroformique.

En placer $1 \mathrm{~cm}^{3}$ dans un tube à essai. Ajouter $\mathrm{X}$ gouttes d'ammoniaque, et $\mathrm{X}$ gouttes d'eau distillée. Agiter et laisser reposer. Quand les deux liquides sont séparés, à l'aide d'un tube très effilé, 
faire arriver $0 \mathrm{~cm}^{3} 5$ environ de réactif de Rupeau-Le-Mithouard (1). Le réactif traverse le chloroforme et donne, au contact du liquide aqueux ammoniacal, un anneau rouge caractéristique de la présence de l'acide picrique.

Nous avons observé qu'avec le filtrat débarrassé du lactose, la diazoréaction de Derrien est négative. Cela prouve que l'acide picrique est éliminé par le lait en nature, et non sous forme d'acide picramique, comme dans l'urine.

Toutefois, on peut, si on veut, effectuer la diazoréaction, à condition de réduire auparavant l'acide ṕicrique. Pour cela :

Dans une fiole conique, verser les $50 \mathrm{~cm}^{3}$ de filtrat restant. Ajouter $3 \mathrm{gr}$. de poudre de zinc, $6 \mathrm{~cm}^{3}$ de lessive de soude et chauffer pendant quelques minutes sur un bain-marie.

Après refroidissement et filtration, neutraliser exactement par l'acide sulfurique.

Prélever $20 \mathrm{~cm}^{3}$ de liquide, filtré si besoin, ajouter 11 gouttes de solution à $1 \%$ de nitrite de sodium et $X$ gouttes d'acide sulfurique dilué au quart; agiter. Ajouter ensuite XXX gouttes d'ammoniaque saturée de $\beta$-naphtol au moment du besoin; mélanger et agiter avec de l'éther. Ce dernier se sépare coloré en violet.

\section{RECHERCHES SUR LE KÉFIR (2)}

Le kéfir est une boisson d'origine caucasienne, préparée avec du lait de chèvre, de vache ou de brebis, fermenté sous l'influence d'un certain nombre de microorganismes qui coagulent finement la caséine et transforment le lactose en acide lactique, alcool et gaz carbonique. On obtient ainsi un liquide blanc, mousseux, de consistance plus ou moins crémeuse, d'odeur et de saveur agréables.

D'après SkLomowSKI, ]'origine du kéfir serait cette boisson connue sous le nom d' (ayran» préparée depuis fort longtemps par les montagnards des pentes-nord du Caucase. Ils obtenaient l'ayran en laissant séjourner pendant un certain temps le lait de leurs bêtes dans des outres réservées à cet usage, et qui n'étaient jamais lavées ni nettoyées ; jusqu'au jour où certains d'entre eux découvrirent dans ces outres des grains blanchâtres, disposés en grappes sur les

(1) Réactif :

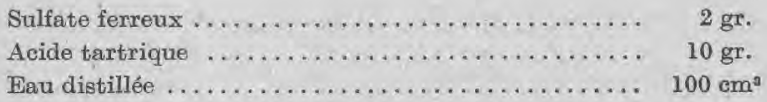

(2) Boris Bourounofw, Thèse de Doct. vét., Lyon, 1933. Travail du Laboratoire de Chimio de l'Ecole vétérinaire de Lyon. Analysé par R. Vurutume, ohef de travaux de chimie à 1'Ecole vétérinaire de Lyon. 